\title{
Author Correction: Hot carrier photovoltaics in van der Waals heterostructures
}

Kamal Kumar Paul, Ji-Hee Kim and Young Hee Lee (D)

Correction to: Nature Reviews Physics https://doi.org/10.1038/s42254-020-00272-4, published online 29 January 2021.

In the original version of this article, the author, Kamal Kumar Paul appeared as only affiliated with the IBS Center for Integrated Nanostructure Physics (CINAP), Institute for Basic Science, Suwon, South Korea. The author is also affiliated with the Department of Energy Science, Sungkyunkwan University, Suwon, South Korea. This error has now been corrected in the HTML and PDF versions of the article.

https://doi.org/10.1038/s42254-021-00377-4 I Published online 31 August 2021

(๑) Springer Nature Limited 2021 